\title{
CORRECTION OF LINEAR COUPLING ON THE BASIS OF RESPONSE MATRIX MODELLING AND X-RAY PINHOLE MEASUREMENT
}

\author{
R. Nagaoka, P. Elleaume, L. Farvacque, J.M. Filhol, ESRF, Grenoble, France
}

\section{Abstract}

A new attempt is made to model a linearly coupled machine using the measured coupled-orbit response. Rather than seeking for the skew errors of the existing magnets, the aim is to obtain an effective skew error distribution that consistently describes the measured coupling characteristics. Such a solution is actually found, which is assured by the simulation that the measured orbit cross-talk provides sufficiently accurate information on the locally integrated skew errors in the ESRF configuration. To pursue the limit of correction, an optimisation of the skew corrector position is then made with respect to the obtained error distribution, with which a large reduction by nearly an order of magnitude is achieved on the model as compared to the standard correction. The actual steps in realising the lowest coupling values on the real machine with additional correctors are described. In the regime where the magnitude of the orbit cross-talk is already too small to be measured, the X-ray pinholes are utilised, which are capable of measuring the vertical emittance down to several picometres.

\section{INTRODUCTION}

The correction of linear coupling is of great importance at the ESRF [1], as the coupling is inversely proportional to the brilliance, the principal machine performance. The coupling at the ESRF is corrected to better than $1 \%$ for daily operation from the initial value of larger than $10 \%$ with 16 skew correctors. Focusing upon the fact that a non-zero off-diagonal orbit response matrix, otherwise called the orbit cross-talk, results from the linear coupling, the correction starts from minimising the orbit cross-talk to a certain degree, which provides a good base for the subsequent resonance compensations. This motivated us to develop an improved minimisation of the orbit cross-talk to further reduce the coupling.

In the meanwhile, an accurate calibration of quadrupoles was carried out using the diagonal part of the response matrix [2], which triggered our second motivation to solve for the skew errors from the off-diagonal matrix, as a natural extension of the work on the diagonal matrix. In the coupled equations of the orbit response due to a steerer excitation, the skew strengths appear linearly in the dipolar terms for the orthogonal orbit. A vertical orbit shift $\Delta z_{C O}$ due to $j$ th horizontal steerer by $\Delta \theta_{H j}$, can be represented for example in the matrix notation as

$$
\frac{\Delta z_{C O}}{\Delta \theta_{H j}}=\mathbf{R}_{i k}^{(V)} \bullet \mathbf{R}_{k j}^{(H)} \bullet \mathbf{a}
$$

where a represents an array of skew errors, the positions of which are defined by the model. $R^{(H)}{ }_{k j}$ and $R^{(V)}{ }_{i k}$ are respectively, the horizontal response matrix from steerer $j$ to skew quadrupole $k$, and the vertical from skew quadrupole $k$ to BPM $i$. It is assumed that they are known accurately from the diagonal matrix analysis. The skew errors can thus be solved via the matrix inversion (e.g. SVD method).

\section{MODELLING OF A LINEARLY COUPLED MACHINE [3]}

\subsection{Simulation}

Minimisation of the orbit cross-talk is found to be made effectively with a general least square method, readily bringing the rms of the cross-talk orbit down to several microns. A noteworthy finding resulted, however, that the reduced orbit cross-talks do not necessarily produce smaller couplings. The relation turns out to depend sensitively on the steerer-BPM-skew corrector configuration. In the case of ESRF, the best solutions for the coupling even increase the orbit cross-talk.



Figure 1: Locally integrated skew strengths (every 10 meters) between the original and reconstructed machines.

Although the orbit cross-talks and the betatron motions satisfy the same coupled equations, the fact that the former are special periodic solutions is suspected to be the underlying reason for this non-trivial correlation. With respect to the solution of Eq. 1 for the specific ESRF configuration, we find that there is not a good enough resolution to identify the individual tilt errors of the quadrupoles. However, locally integrated strengths can be well reproduced (Fig. 1), which describe the coupling of 
the original machine locally and globally in a satisfactory manner. In contrast to the work made on the same principle [4], the importance of building an effective skew distribution may therefore be stressed, which also conforms with the fact that the skews errors due to vertical orbit offsets in the sextupoles are comparable to the quadrupole tilts for the ESRF machine.

\subsection{Application to the Real Machine}

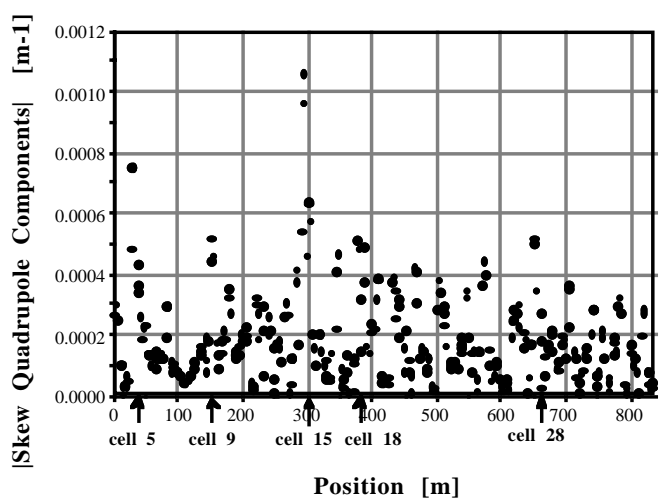

Figure 2: Obtained skew distribution of the uncorrected coupling.

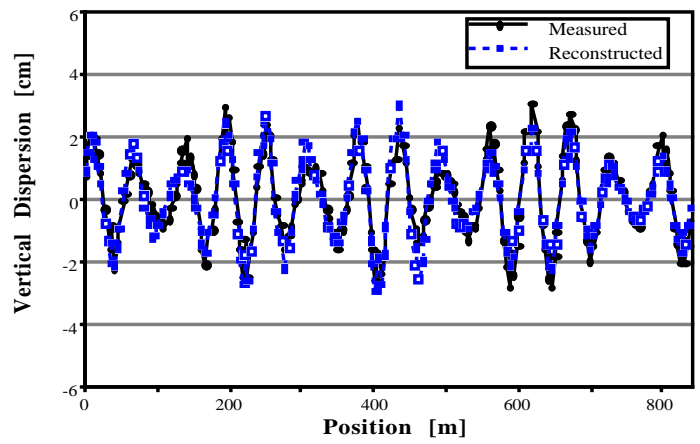

Figure 3: Predicted vertical dispersion of the uncorrected coupling, in comparison with the measured one.

With the measured matrices, the effect of averaging turns out to be particularly important in removing the involved imperfections. Equation 1 is solved with the SVD method for a pair of steerers belonging to the same family, optics wise, whose skew distribution is averaged over the entire pairs. Searching the best number of eigenvectors is found to be equally important. The measured coupled orbits are reproduced typically to few microns rms. The resultant skew distribution of the machine without coupling correction exhibits several localised peaks (Fig. 2). It reproduces well the measured vertical dispersion (Fig. 3), as well as the tune separation around the coupling resonance (Fig. 4), i.e. the two major coupling observables. Most computations were made with 10 skew flags per cell. Although valid solutions can be obtained with a smaller number of flags, it results in increasing the magnitude of the individual skew strengths.

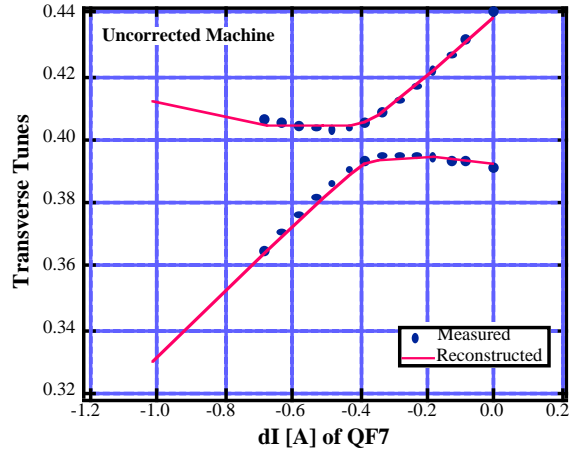

Figure 4: Predicted tune separation of the uncorrected coupling, in comparison with the measured one.

\section{COUPLING CORRECTION}

Having obtained the effective error skew distribution of the ESRF machine, one can attempt to correct the coupling on the model. The resulting skew corrector strengths agree well with those actually obtained from the standard corrections on the field.

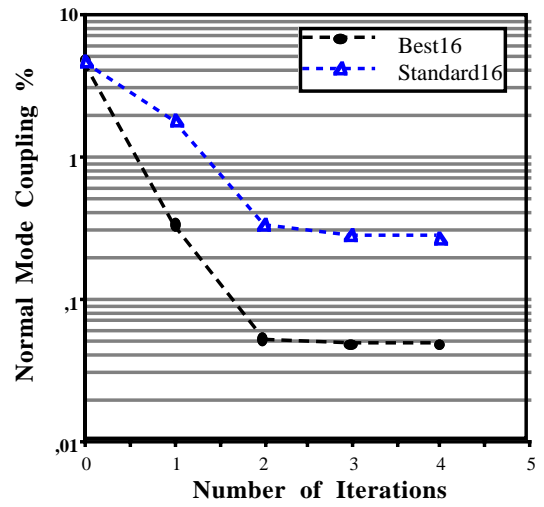

Figure 5: Correction efficiency of the most effective correctors, in comparison with the standard ones.

As an useful application of the model, a search was made for the most effective corrector positions with respect to the skew errors. Placing 96 skew correctors around the model machine at allowed locations, minimisation of the normal mode coupling was made with the best corrector method, taking account of the correlation among the selected correctors. A large reduction to $\sim 0.05 \%$ was obtained on the model coupling with 16 selected correctors, as compared to $\sim 0.3 \%$ reached with the standard correctors (Fig. 5). Interestingly, the orbit cross talk is simultaneously suppressed in the optimised correction. Figure 6 summarises the relation between the coupling and the orbit cross-talk for the three characteristic coupling states: 1) Uncorrected. Best corrected with 2) the existing correctors, and with 3 ) the most effective correctors. 


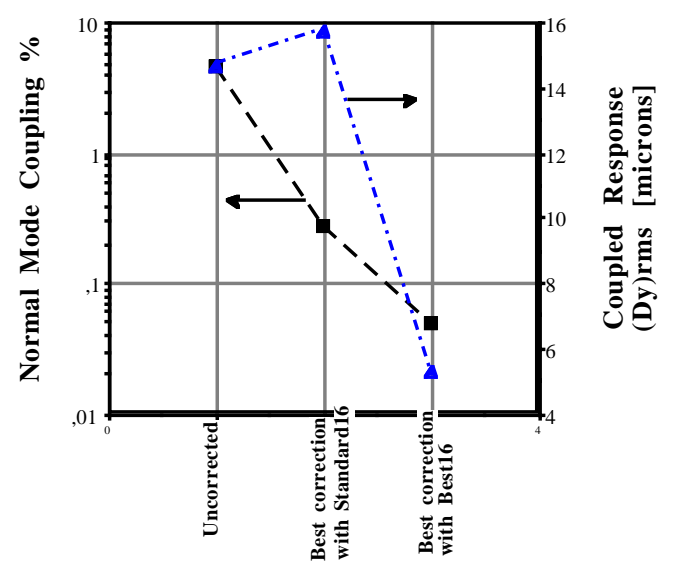

Figure 6: Relation between the coupling and the orbit cross-talk for three different correction states described in the text.

16 additional skew correctors were installed accordingly in the ring. Anticipating unavoidable discrepancies between the model and the real machine as well as the reduction of the orbit cross-talk which would prevent from iterating the response matrix analysis, an alternative correction method was prepared. The developed scheme minimises the vertical beam sizes measured with the X-ray pinhole cameras at two different locations in the ring. Utilising the bending magnet radiation, the pinhole set-ups are capable of measuring the vertical emittances down to 5 pm.rad. Details are found in Ref. 5. Simulations show that with the vertical dispersion included with a proper weighting, the minimisation of the vertical beam sizes at the two pinhole locations manages to reduce the coupling.

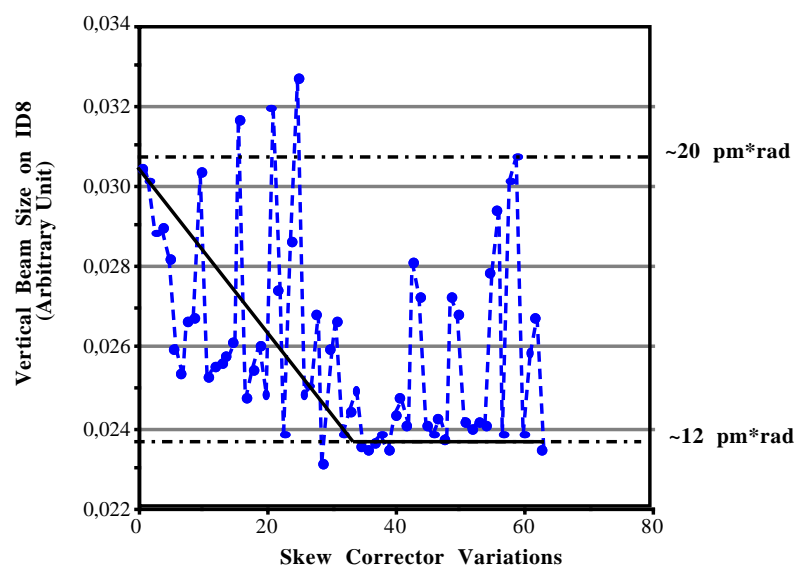

Figure 7: Reduction of the measured vertical beam size with the number of steps in the empirical correction.

Applying the model solutions to the machine, the coupling expectedly reached a level lower than the standard value. The empirical minimisation of the measured vertical beam sizes further reduced successfully the vertical emittances down to $12 \mathrm{pm} \cdot \mathrm{rad}(0.3 \%$ coupling $)$. In accordance with the prediction, the measured orbit cross talk was significantly suppressed (the rms is $5 \mu \mathrm{m}$ against
$4 \mu \mathrm{m}$ of predicted). Together with the vertical dispersion which is reduced to $\sim 0.3 \mathrm{~cm} \mathrm{rms}$ (instead of $0.15 \mathrm{~cm}$ predicted), the reached setting is expected to be in the vicinity of the best point.
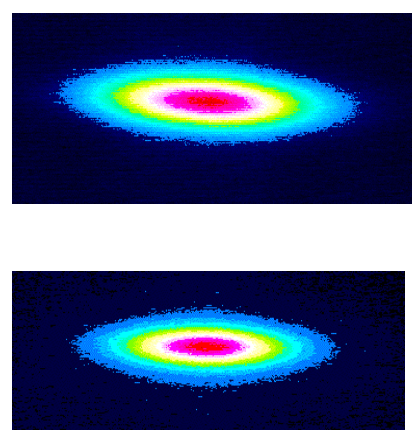

Figure 8: The two beam images of the $\mathrm{X}$-ray pinholes at the lowest coupling achieved $\left(\varepsilon_{\mathrm{V}} \sim 12 \mathrm{pm} \cdot \mathrm{rad}\right)$. Note that the beta values at the source points are approximately $2.7 \mathrm{~m}$ horizontally and $35 \mathrm{~m}$ vertically.

\section{CONCLUSION}

It was demonstrated that from the coupled-response matrix (orbit cross-talk), sufficiently accurate information can be extracted on the source of the linear coupling, namely the effective error skew distribution. The present approach therefore has the great advantage of understanding quantitatively the local details of the coupling of the machine, which is of particular importance for synchrotron light sources. The derived model was successfully utilised to perform the most effective correction by optimising the corrector locations. Starting from the model solution, minimisation of two vertical beam sizes measured with X-ray pinholes managed to lower the coupling to $\sim 0.3 \%$ from the standard value of $\sim 0.8 \%$. The reason for the remaining discrepancy to the predicted coupling is to be investigated.

\section{ACKNOWLEDGEMENT}

The authors would like to express their thanks to all those who supported this work from different groups at the ESRF.

\section{REFERENCES}

[1] J.M. Filhol, L. Hardy and U. Weinrich, ESRF Status Report, this conference.

[2] L. Farvacque and R. Nagaoka, "Calibration of Quadrupole Magnets via Response Matrix Modelling", EPAC98, Stockholm, June 1998.

[3] R. Nagaoka, "Modelling of a Linearly Coupled Machine Using the Coupled-Response Matrix”, EPAC98, Stockholm, June 1998.

[4] J. Safranek, "Experimental Determination of Storage Ring Optics Using Orbit Response Measurements", Nucl. Instr. And Meth., A388 (1997) 27.

[5] P. Elleaume, C. Fortgang, C. Penel and E. Tarazona, "Measuring Beam Sizes and Ultra-Small Electron Emittances Using an X-ray Pinhole Camera",

J. Synchrotron Rad., 2 (1995) 209. 